\title{
Resveratrol protects primary cortical neuron cultures from transient oxygen-glucose deprivation by inhibiting MMP-9
}

\author{
DAKUAN GAO*, TAO HUANG* ${ }^{*}$ XIAOFAN JIANG, SHIJIE HU, LEI ZHANG and ZHOU FEI \\ Department of Neurosurgery, Xijing Hospital, Fourth Military Medical University, Xi'an, Shaanxi 710032, P.R. China
}

Received October 2, 2013; Accepted February 18, 2014

DOI: $10.3892 / \mathrm{mmr} .2014 .2086$

\begin{abstract}
It was recently shown that resveratrol exerts neuroprotective effects against cerebral ischemia in mice. The aim of the present study was to further confirm these effects in in vitro primary cortical neuron cultures with transient oxygen-glucose deprivation (OGD), and to investigate whether these effects are due to the inhibition of matrix metalloproteinase-9 (MMP-9) and of cell apoptosis. Neuronal primary cultures of cerebral cortex were prepared from BALB/c mice embryos (13-15 days). Cells from 14- to 16-day cultures were subjected to OGD for $3 \mathrm{~h}$, followed by $21 \mathrm{~h}$ of reoxygenation to simulate transient ischemia. Different doses of resveratrol were added into the culture medium during the simulation of transient ischemia. The effect of the extracellular signal-regulated kinase (ERK) inhibitor U0126 was studied by adding U0126 $(5 \mu \mathrm{g} / \mu \mathrm{l}, 4 \mu \mathrm{l})$ into the culture medium during transient ischemia; as a control, we used treatment of cells with $50 \mu \mathrm{M}$ of resveratrol. Cell viability was investigated using the 3-[4,5-dimethylthiazol-2-yl]-2,5 diphenyl tetrazolium bromide (MTT) reduction assay. Cell apoptosis was assessed by flow cytometry. The effects of resveratrol on the expression of MMP-9 were analyzed by western blotting and reverse transcription-polymerase chain reaction (RT-PCR), while the levels of ERK, phosphorylated (p)-ERK, cleaved caspase-3, Bax and Bcl-2 were measured by western blotting. The results of the MTT assay showed that cell viability is significantly reduced by transient OGD. OGD induced cell apoptosis, the expression of Bax and the activation of caspase-3 and ERK, inhibited the expression of $\mathrm{Bcl}-2$ and increased the expression of $M M P-9$, while these effects were reversed by treatment with resveratrol. The therapeutic efficacy of resveratrol was shown to be dose-dependent, with the most suitable dose
\end{abstract}

Correspondence to: Dr Zhou Fei, Department of Neurosurgery, Xijing Hospital, Fourth Military Medical University, 169 Changle West Road, Xi'an, Shaanxi 710032, P.R. China

E-mail: zhoufeishaanxi@163.com

*Contributed equally

Key words: resveratrol, neuron, oxygen-glucose deprivation, ERK, MMP-9, apoptosis range determined at 50-100 $\mu \mathrm{M}$. Treatment with U0126 inhibited MMP-9 and Bax expression and caspase-3 activation, while it further promoted the expression of the anti-apoptotic molecule Bcl-2, suggesting that resveratrol inhibits MMP-9 expression and cell apoptosis by attenuating the activation of ERK1/2. In conclusion, OGD can induce apoptosis through canonical apoptotic signals and by regulating the expression of MMP-9; the anti-apoptotic activity of resveratrol and its inhibitory effect on MMP-9 expression contribute in the reduced activation of ERK.

\section{Introduction}

The brain is the most vulnerable organ to ischemic infringement. Neuronal apoptosis is involved in the pathophysiology of brain ischemia and reperfusion injury. Therefore, protection from abnormally increased neuronal apoptosis is expected to be beneficial to therapy.

Resveratrol (trans-3,4',5-trihydroxystilbene) is a natural phytoalexin found in grapes and other plants that has anticancer, neuroprotective and anti-inflammatory effects (1-3). In addition, resveratrol was found to have anti-oxidant and chemopreventive properties (4). Resveratrol was also shown to reduce the size of cerebral infarction in mice $(5,6)$ and demonstrated protective effects against brain injury induced by ischemia-reperfusion in gerbils (6). Its beneficial neuroprotective effect may be due to its inhibitory effect on platelet aggregation, its vasodilating effect, its anti-oxidant activity or the combination of these (5-9).

Inappropriate expression of matrix metalloproteinases (MMPs) is thought to contribute to the pathogenesis of various conditions, such as arthritis, periodontal disease, atherosclerosis, cancer and ischemia $(10,11)$. Among the known MMPs, gelatinase-B (MMP-9) is a key enzyme for the degradation of type IV collagen, which is a major component of the basement membrane (12-14). MMP-9 is expressed at a low level in the brain of healthy adult rats (15). MMP-9 is a key regulator of apoptosis of hypertrophic chondrocytes, and null mutations in the gene can delay apoptosis (16). A previous study from our group demonstrated that cerebral ischemia-reperfusion induces MMP-9 expression in mice (17). However, whether resveratrol is the MMP inhibitor that is associated with cerebral ischemia remains unknown.

In the present study, the effects of resveratrol on injury induced by oxygen-glucose deprivation (OGD), including 
neuronal apoptosis and changes in the expression of MMP-9, were examined in primary cortical neuron cultures. Potentially relevant protective mechanisms were also investigated.

\section{Materials and methods}

Mouse cortical cultures. The present study was approved by the ethics committee of Xijing Hospital (Xi'an, Shaanxi, China). Cultures of cortical neurons from mice were prepared as previously described (18). Timed-pregnant (13-15 days) $\mathrm{BALB} / \mathrm{c}$ mice were anesthetized with halothane and sacrificed by cervical dislocation. Following dissection of the cortical region of the fetal brain using a somatotype microscope (Beijing Taike Instrument Co., Ltd., Beijing, China), cortical neurons were dispersed by trituration and digestion in $0.25 \%$ trypsin (Sigma-Aldrich, St. Louis, MO, USA) for $30 \mathrm{~min}$ at $37^{\circ} \mathrm{C}$. Then, the cell suspension was centrifuged at $250 \mathrm{x} \mathrm{g}$ for $5 \mathrm{~min}$ at $4^{\circ} \mathrm{C}$ and resuspended in dissociating medium [Dulbecco's modified Eagle's medium with 10\% fetal bovine serum (Gibco, Carlsbad, CA, USA), 10 mM HEPES, $44 \mathrm{mM}$ glucose and $2 \mathrm{mM}$ L-glutamine (all from Sigma-Aldrich)]. Cells $\left(1 \times 10^{6}\right.$ cells $\left./ \mathrm{ml}\right)$ were plated on poly-L-lysine-coated culture plates. After $24 \mathrm{~h}$, the medium was replaced with Neurobasal medium consisting of 2\% B27 supplement, $0.5 \mathrm{mM}$ L-glutamine and $25 \mu \mathrm{M}$ glutamate (all from Sigma-Aldrich) to minimize glial growth. At 7 days of growth, one-half of the medium was replaced with fresh Neurobasal medium. Experiments were performed on cells from 14- to 16-day cultures.

Simulation of ischemia and reperfusion in vitro. To induce OGD, the medium was removed from the cultures and stored. Cultures were rinsed twice with phosphate-buffered saline (PBS) and low-glucose Dulbecco's modified Eagle's medium (Gibco) with 2\% B27 was added. Cultures were then transferred to a humidified chamber kept in a $37^{\circ} \mathrm{C}$ incubator, and subjected to an anaerobic environment of $95 \% \mathrm{~N}_{2}-5 \% \mathrm{CO}_{2}$ for $3 \mathrm{~h}$. Oxygen concentration was maintained at $0.5-1.0 \%$, as monitored by an oxygen analyzer (MSA Medical, Pittsburgh, PA, USA), throughout the experiment. OGD was terminated by adding the stored medium to the cultures, followed by incubation at $37^{\circ} \mathrm{C}$ in a $5 \% \mathrm{CO}_{2}$ atmosphere for $21 \mathrm{~h}$ to allow reoxygenation.

Resveratrol and U0126 treatment. A stock solution (100 mM) of resveratrol (Sigma-Aldrich) was prepared in dimethylsulfoxide (DMSO; Sigma-Aldrich) and stored at $-20^{\circ} \mathrm{C}$. For treatment, resveratrol was diluted in PBS and added to the cultures in order to obtain the desired final concentrations $(10,25,50$ and $100 \mu \mathrm{M})$. U0126 (Sigma-Aldrich) is a specific extracellular signal-regulated kinase (ERK) inhibitor. U0126 ( $5 \mu \mathrm{g} / \mu \mathrm{l}, 4 \mu \mathrm{l}$, in DMSO) was added to the cultures $15 \mathrm{~min}$ prior to resveratrol treatment at a final concentration of $50 \mu \mathrm{M}$. Untreated cultures (vehicle controls for treatment with resveratrol or U0126) received the same amount of the carrier solvent (0.1\% DMSO). The duration of the treatment was from OGD until the end of the experiment.

Cell viability assay. The assay is based on measuring the reduction, by dehydrogenases of metabolically active cells, of the MTT tetrazolium (3-(4,5-dimethylthiazolyl-2-)-2,5-diphenyltetrazolium bromide; Sigma-Aldrich) that is yellow, to a purple formazan. The intracellular formazan can be solubilized and quantified by spectrophotometry. Cells from neuronal primary cultures were grown on 96 -well plates at a density of $2 \times 10^{5}$ cells $/ \mathrm{cm}^{2}$. At 14 days of growth, cells were subjected to OGD and reoxygenation, with different concentrations of resveratrol added into the medium. After $21 \mathrm{~h}$ of reoxygenation, MTT was added to the cells at a final concentration of $0.5 \mathrm{mg} / \mathrm{ml}$ and the plates were incubated for $4 \mathrm{~h}$ at $37^{\circ} \mathrm{C}$. The insoluble formazan product was then precipitated by centrifugation, the supernatant removed, and the crystals were dissolved in $100 \mu \mathrm{l}$ DMSO. Absorbance at $570 \mathrm{~nm}$ was measured using a Bio-Rad microplate reader (Bio-Rad, Hercules, CA, USA). The ratio of the absorbance of treated cells to that of the control cells was calculated and used to represent the percentage of growth inhibition.

Apoptosis assay. Neuronal apoptosis was assayed by flow cytometry using the Annexin V-FITC Apoptosis Detection kit (Sigma-Aldrich) as described previously (19). Briefly, $1 \times 10^{6}$ single cells per sample were collected after $3 \mathrm{~h}$ of OGD and the subsequent 21-h reoxygenation treatment and were washed twice with PBS buffer. Following addition of Annexin V-FITC and incubation for $10 \mathrm{~min}$ at room temperature in the dark, the cells were washed and resuspended, and propidium iodide was added to a final concentration of $1 \mathrm{mg} / \mathrm{l}$. Stained cells were analyzed using a FACSCalibur cytometer (BD Biosciences, Mountain View, CA, USA).

Western blot analysis. Following treatment, cells were incubated in lysis buffer (50 mM Tris-HCl, pH 7.6; $150 \mathrm{mM} \mathrm{NaCl}$; $0.1 \%$ polyoxyethyleneglycol dodecyl ether; $0.1 \%$ deoxycholic acid; $10 \mu \mathrm{g} / \mathrm{ml}$ leupetin; $2 \mu \mathrm{g} / \mathrm{ml}$ aprotinin; and $1 \mathrm{mM}$ phenyl methane sulfonyl fluoride) on ice. Following centrifugation, the supernatant was collected and total protein concentrations were determined using the Bradford assay (Bio-Rad). Equal amounts (30 $\mu \mathrm{g}$ in $10 \mu \mathrm{l}$ ) of total protein extract were mixed with $2 \mathrm{X}$ sample buffer (125 mM Tris pH 6.8, 25\% (v/v) glycerol, 4\% SDS, $10 \% \beta$-mercaptoethanol), and separated by SDS-PAGE. Next, proteins were transferred onto a nitrocellulose membrane using a commercial semi-dry blotting apparatus (Bio-Rad, Richmond, CA, USA). The membrane was blocked overnight at $4^{\circ} \mathrm{C}$ by adding $10 \%$ non-fat dry milk in Tris-buffered saline (pH 7.4) supplemented with $0.1 \%$ Tween-20 (TBS-T). Then, the membrane was incubated with the primary antibodies antiMMP-9, - $\beta$-actin, -Bcl-2 and -Bax (Sigma-Aldrich), polyclonal anti-ERK and-phospho (p)-ERK, and monoclonal anti-caspase-3 (Cell Signaling Technology, Inc., Danvers, MA, USA), all of which were diluted in blocking buffer (Tiangen Biotech Co., Ltd., Beijing, China), for $2 \mathrm{~h}$ at room temperature. The primary antibodies targeting MMP-9 and $\beta$-actin were diluted with PBS at 1:1,000 and 1:2,000, respectively. After washing with TBS-T, the membrane was incubated at room temperature for $1 \mathrm{~h}$ with horseradish peroxidase-conjugated secondary antibodies, i.e., anti-rabbit and anti-rat IgG produced in goat and targeting MMP-9 and $\beta$-actin, respectively (Sigma-Aldrich). Detection of the targeted antigens was performed with a standard electrochemical luminescence method (ECL kit; Santa Cruz Biotechnology, Inc., Santa Cruz, CA, USA). 
Reverse transcription-polymerase chain reaction (RT-PCR) analysis. Expression of the MMP-9 gene was examined using RT-PCR. Total RNA was isolated using the TRIzol reagent (Invitrogen Life Technologies, Carlsbad, CA, USA), according to the manufacturer's instructions. Five $\mu \mathrm{g}$ of total RNA were incubated with 100 units of Superscript ${ }^{\mathrm{TM}}$ II reverse transcriptase (Invitrogen Life Technologies), dNTPs (175 $\mu \mathrm{M}), 200 \mathrm{ng}$ oligo(dT), dithiothreitol $(1 \mu \mathrm{M})$ and reaction buffer in a final volume of $20 \mu \mathrm{l}$, at $37^{\circ} \mathrm{C}$ for $60 \mathrm{~min}$. In control reaction mixtures, reverse transcriptase A was omitted in order to determine the amplification of contaminating genomic DNA or cDNA. After denaturation at $94^{\circ} \mathrm{C}$ for $5 \mathrm{~min}, 1 \mu \mathrm{l}$ of cDNA was subjected to PCR. PCR amplifications were performed by incubating in the following three conditions: $1,94^{\circ} \mathrm{C}$ for $30 \mathrm{sec}$; $2,56^{\circ} \mathrm{C}$ for $1 \mathrm{~min} ; 3,72^{\circ} \mathrm{C}$ for $1 \mathrm{~min}$. A total of 35 cycles were performed for the amplification of $M M P-9$ and 30 cycles for $\beta$-actin. The last cycle was followed by $10 \mathrm{~min}$ of elongation at $72^{\circ} \mathrm{C}$. Primer pairs for the specific amplification of $M M P-9$ (based on GenBank accession no. Z27231) and $\beta$-actin (accession no. V01217) from murine cDNA were the following: MMP-9 forward (bp: 835-854), 5'-TAGTGAGAGACT CTACACAG-3' and reverse (bp: 1155-1174), 5'-CCACTTCTT GTCAGTGTCGA-3'; $\beta$-actin forward (bp: 331-354), 5'-AACCCTAAGGCCAACCGTGAAAAG-3' and reverse (bp: 551-571), 5'-TCATGAGGTAGTCTGTCAGGT-3'. The lengths of the MMP-9 and $\beta$-actin amplicons were 340 and 242 bp, respectively. PCR products were visualized on $1.5 \%$ agarose gels stained with ethidium bromide, under a UV transilluminator. Semi-quantitative analysis was conducted using a computerized densitometric imager (Bio-Rad).

Statistical analysis. Results are expressed as mean \pm SD from at least three independent experiments. Statistical analysis was performed using Student's t-tests and one-way analysis of variance. A difference was considered statistically significant at $\mathrm{P}<0.05$ or $\mathrm{P}<0.01$.

\section{Results}

Cell viability assay. The MTT cell viability assay is commonly used to analyze metabolic activity in cells. It measures the reduction of tetrazolium salts facilitated by mitochondrial dehydrogenases. A number of factors such as cell number, cell metabolism and mitochondrial activation influence the outcome of this assay. The number of surviving cells is directly proportional to the level of the formed formazan product. Exposure of the cells to OGD for $3 \mathrm{~h}$ followed by $21 \mathrm{~h}$ of reoxygenation caused a reduction in cell death rate of $\sim 40-50 \%$. Under these conditions, the neuroprotective effect of resveratrol was dose-dependent: $10,25,50$ and $100 \mu \mathrm{M}$ of resveratrol reduced OGD-induced cell death by $30,50,75$ and $78 \%$, respectively. Treatment with a minimal dose of $50 \mu \mathrm{M}$ resveratrol had a significantly beneficial effect on cell viability, with no deleterious side-effects observed at/below $100 \mu \mathrm{M}$ (Fig. 1).

OGD-induced cell apoptosis is attenuated in resveratrol-treated cells. To quantify neuronal apoptosis induced by OGD, flow cytometry was used. As shown in Fig. 2, under normal conditions, the level of neuronal apoptosis is very low

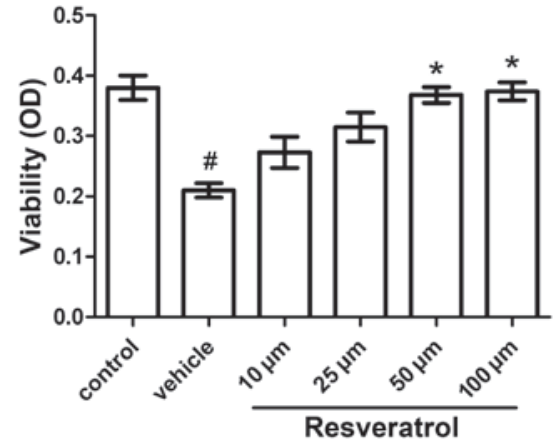

Figure 1. MTT cell viability assay in healthy cells control, oxygen-glucose deprivation (OGD) and vehicle-treated cells (vehicle) and cells subjected to OGD and treated with different doses of resveratrol (10 wells per group). The graph shows mean absorbance at $570 \mathrm{~nm}$, which is an indirect measure of cell viability. The MTT assay results indicated that 50-100 $\mu \mathrm{M}$ resveratrol can significantly protect primary cortical neuron cultures from transient OGD. ${ }^{\#} \mathrm{P}<0.05$ vs. control; ${ }^{*} \mathrm{P}<0.01$ vs. vehicle.

(10.9\%). Following OGD insult, the percentage of apoptosis was markedly increased, reaching $51 \%(\mathrm{P}<0.01)$. Treatment with 25,50 and $100 \mu \mathrm{M}$ of resveratrol prior to OGD significantly decreased the percentage of apoptotic cells to 35.9, 29.6 and $25 \%(\mathrm{P}<0.05)$, respectively; treatment with a lower dose $(10 \mu \mathrm{M})$ of resveratrol also decreased the percentage of apoptotic cells at $43.5 \%$, but this change was not significant ( $P>0.05)$. The vehicle solution (DMSO) had no effect on cell apoptosis induced by OGD $(\mathrm{P}>0.05)$.

Resveratrol influences the apoptotic signaling pathway. To gain further insight into the mechanism by which resveratrol attenuated OGD-induced cell apoptosis, we studied the changes in gene expression of genes associated with apoptosis. Subjection of cells to OGD induced cleavage of caspase-3 (Fig. 3), and 50 or $100 \mu \mathrm{M}$ of resveratrol significantly decreased the level of cleaved caspase-3 $(\mathrm{P}<0.05)$. The expression of the Bax protein was also induced by OGD and decreased by resveratrol (Fig. 3). We further examined the expression of the Bcl-2 protein. OGD decreased Bcl-2 expression, an effect reversed by resveratrol (Fig. 3). These results suggested that resveratrol exerts anti-apoptotic effects via regulating proteins of the canonical apoptotic signaling pathway.

Effect of resveratrol on MMP-9 expression. To gain further insight into the neuroprotective properties of resveratrol, we studied its effect on the expression of MMP-9. In western blot analysis, the 105-kDa latent form of MMP-9 was detected in all samples. Exposure of the cells to OGD for $3 \mathrm{~h}$ followed by $21 \mathrm{~h}$ of reoxygenation induced a significant increase in expression of MMP-9 compared to the control; this elevation in the protein level of MMP-9 was reverted by resveratrol treatment in a dose-dependent manner. Under simulated ischemic conditions, cells treated with $25 \mu \mathrm{M}$ resveratrol had a lower level of MMP-9 compared to cells treated with the carrier solvent solution (vehicle), while even lower levels of MMP-9 were detected in cells treated with 50 and $100 \mu \mathrm{M}$ resveratrol. However, the amount of detected protein was not significantly different between the two latter treatments. The level of the $42 \mathrm{kDa}$ protein $\beta$-actin was used as an internal control and did not significantly change among samples (Fig. 4). 
A
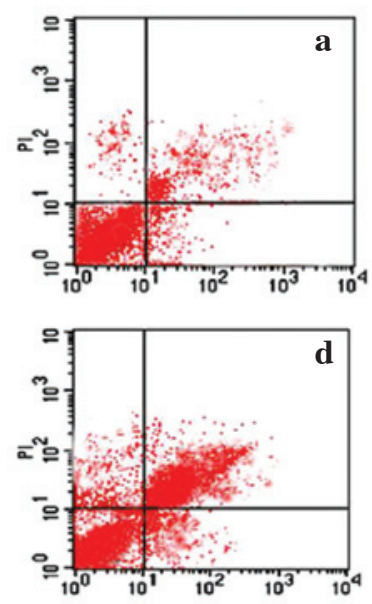
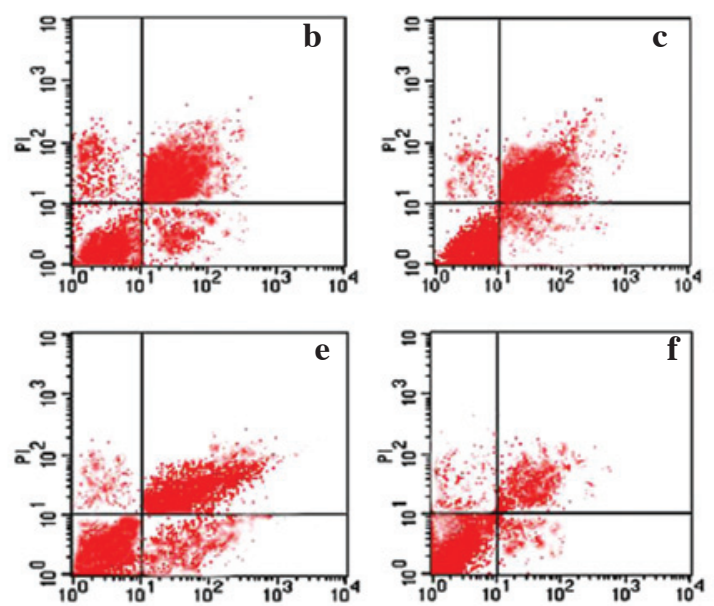

B

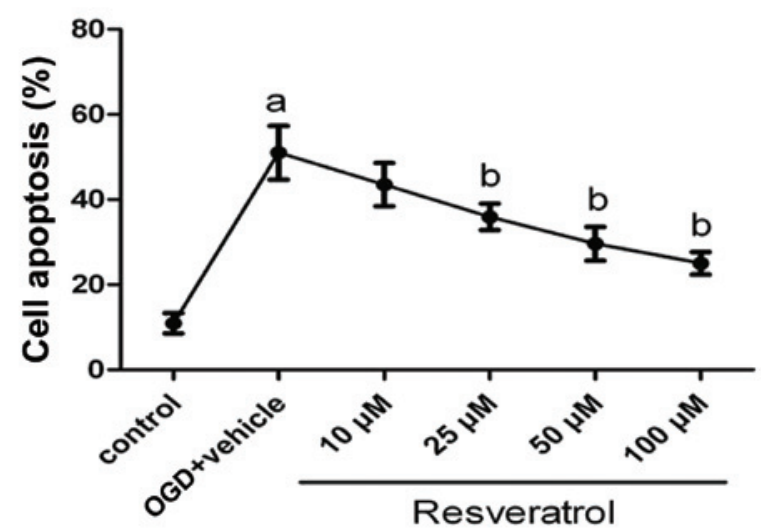

Figure 2. Effect of resveratrol on oxygen-glucose deprivation (OGD)-induced apoptosis in cortical neuron cultures, as measured by flow cytometry. (A) Scatterplots of apoptosis: a) control; b) OGD+vehicle; c) OGD+10 $\mu \mathrm{M}$ Res; d) OGD $+25 \mu \mathrm{M}$ Res; e) OGD+50 $\mu \mathrm{M}$ Res; and f) OGD+100 $\mu \mathrm{M}$ Res. (B) Quantification of cell apoptosis rate data (mean $\pm \mathrm{SD}, \mathrm{n}=5$ ). ${ }^{\mathrm{a}} \mathrm{P}<0.01 \mathrm{vs}$. control; ${ }^{\mathrm{b}} \mathrm{P}<0.05$ vs. OGD+vehicle. Res, resveratrol.

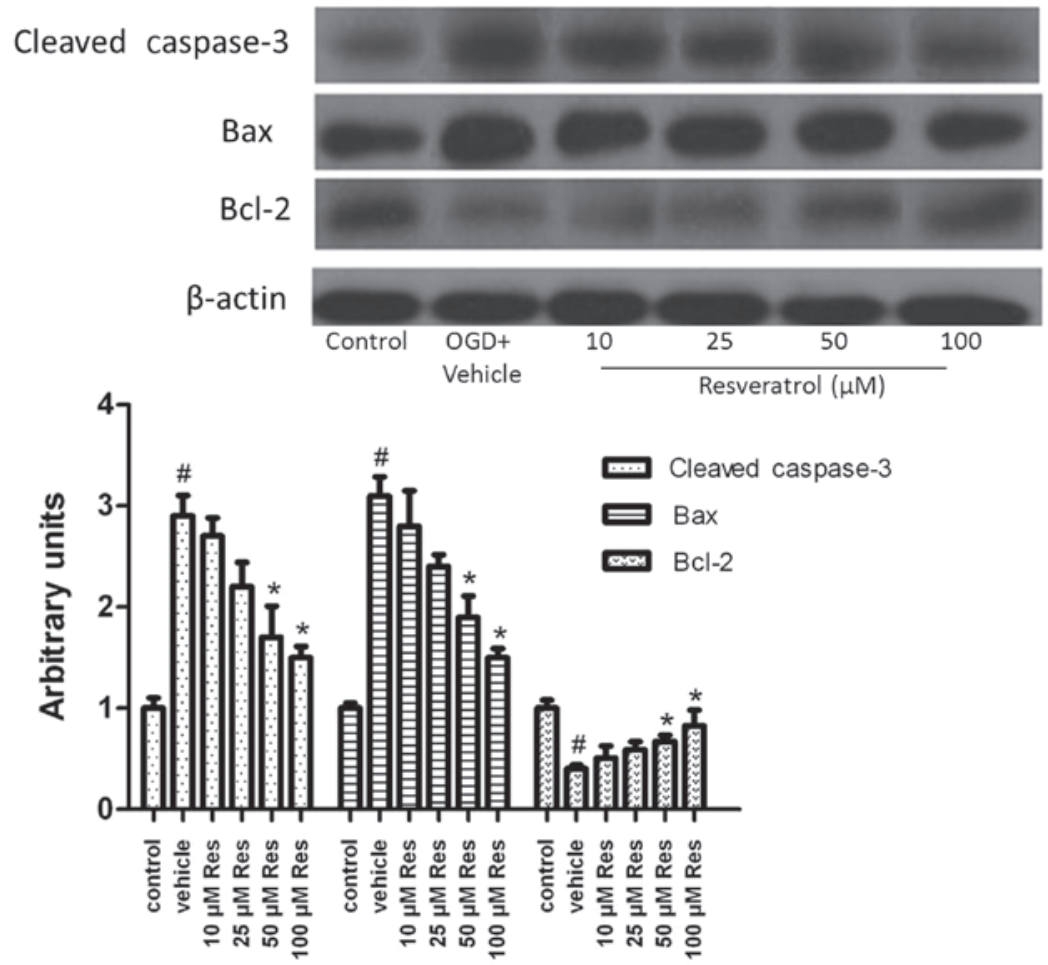

Figure 3. Effect of resveratrol on transient oxygen-glucose deprivation (OGD)-induced apoptotic signaling. The protein levels of cleaved caspase-3, Bcl-2 and Bax were determined using western blotting and the corresponding antibodies. $\beta$-actin was used as an internal control. Each blot is representative of three independent experiments. " $\mathrm{P}<0.05$ vs. control; " $\mathrm{P}<0.05$ vs. OGD+vehicle (vehicle). Res, resveratrol. 

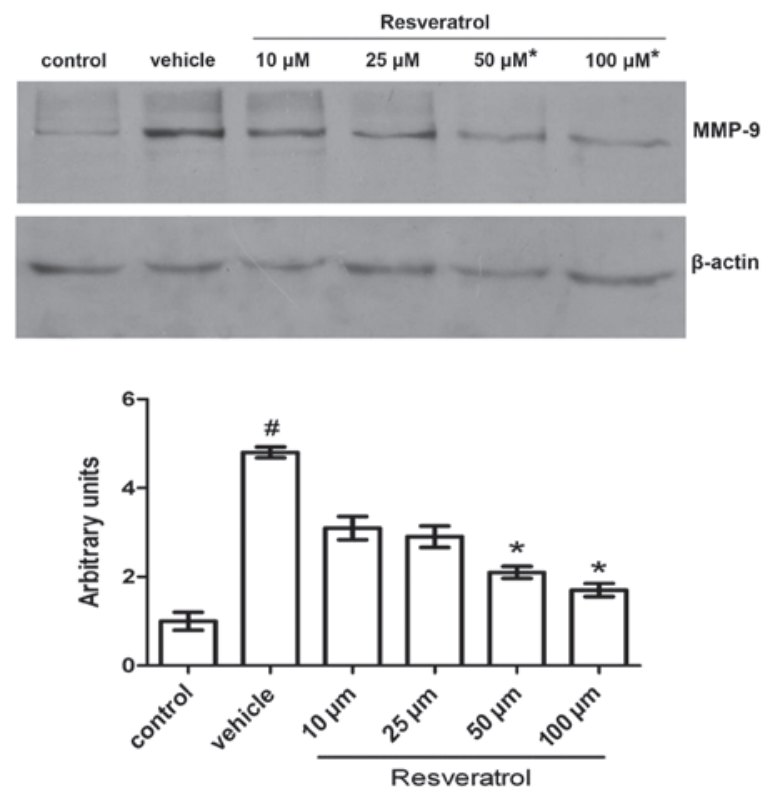

Figure 4. Western blot analysis of the matrix metalloproteinase-9 (MMP-9) level demonstrates upregulation of the protein following transient oxygen-glucose deprivation (OGD). However, the increase in MMP-9 expression was inhibited by resveratrol treatment in a dose-dependent manner. The MMP-9 protein was mainly detected at $105 \mathrm{kDa}$. Mouse $\beta$-actin, $\sim 2 \mathrm{kDa}$, was used as the internal standard. " $\mathrm{P}<0.05$ vs. control; " $\mathrm{P}<0.01$ vs. OGD+vehicle (vehicle).
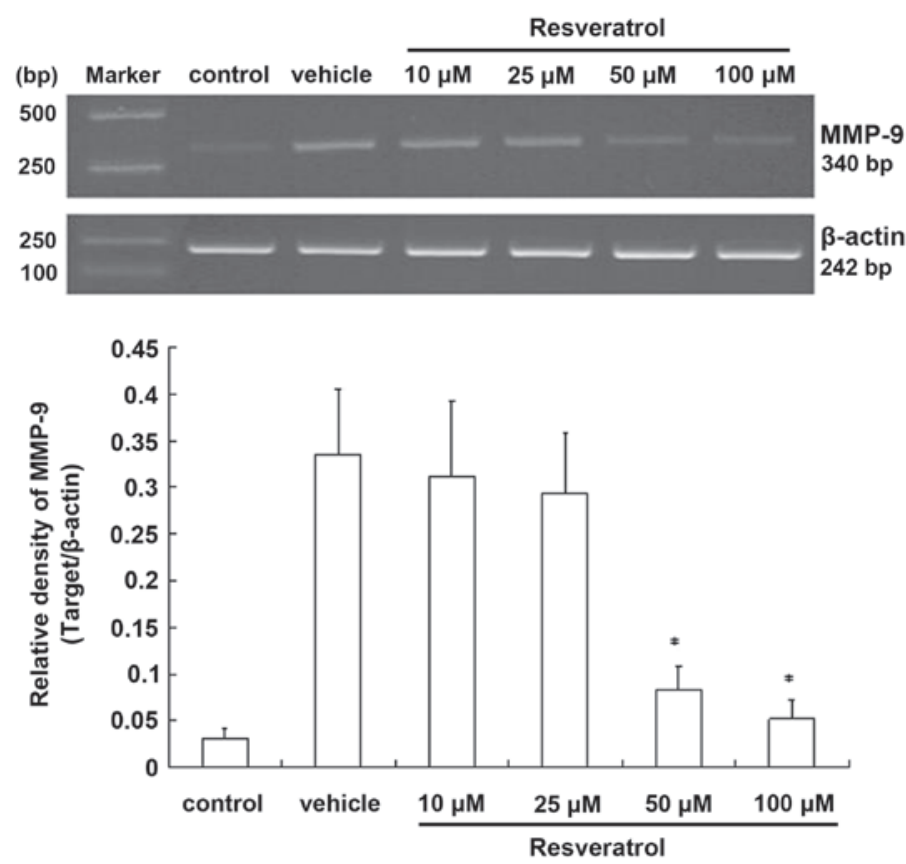

Figure 5. Results of the RT-PCR assay for the matrix metalloproteinase-9 (MMP-9) mRNA show that high doses of resveratrol (50-100 $\mu \mathrm{M})$ significantly decrease the high levels of MMP-9. " $\mathrm{P}<0.01$ vs. transient oxygen-glucose deprivation+vehicle (vehicle). Mouse $\beta$-actin was used as the internal standard. The sizes of the MMP-9 and $\beta$-actin amplicons were 340 and 242 bp respectively. The DNA marker is shown on the left.

To determine whether resveratrol is involved in the regulation of MMP-9 at the mRNA level, we carried out semi-quantitative RT-PCR experiments. In cells exposed to OGD for $3 \mathrm{~h}$ followed by $21 \mathrm{~h}$ of reoxygenation, MMP-9 was prominently expressed, while almost no mRNAs were detected in the control. However, in the resveratrol-treated cells, the level of MMP-9 mRNA was reduced compared to the OGD group, with the reduction being statistically significant at higher doses. By contrast, the $\beta$-actin mRNA level remained unchanged (Fig. 5). Taken together, these results indicated that resveratrol may be involved in the regulation of the expression of the MMP-9 gene. The observed changes in the level of the $M M P-9$ mRNA evaluated by RT-PCR demonstrated that resveratrol suppresses transient OGD-induced expression of $M M P-9$ by inhibiting the gene's transcription.

Resveratrol influences the activation of ERK. ERK plays a crucial role in almost all cell functions; ERK activity mediates distinct antiproliferative events, for example in apoptosis (20). Since the phosphorylated form of the protein is the active form, 

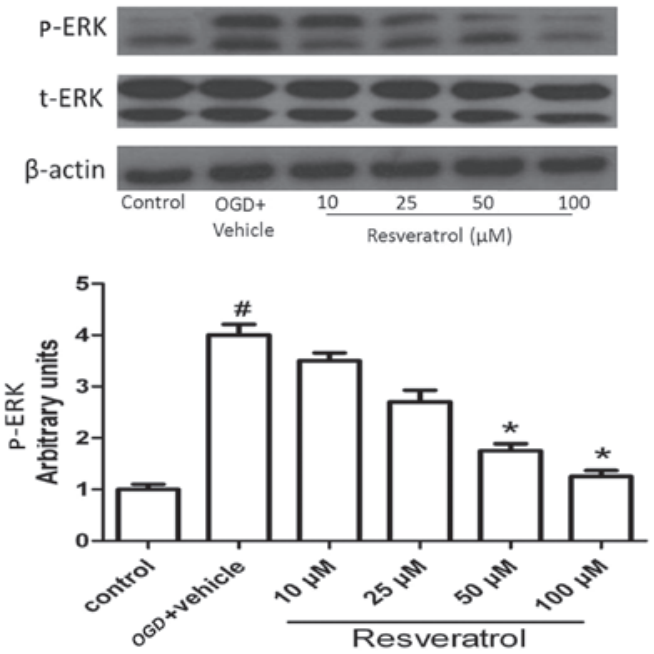

Figure 6. Effect of resveratrol on the oxygen-glucose deprivation (OGD)-induced activation of extracellular signal-regulated kinase (ERK). The protein levels of total ( $t$ )-ERK and phosphorylated (p)-ERK were determined using western blotting and the corresponding antibodies. $\beta$-actin was used as an internal control. Each blot is representative of three independent experiments. ${ }^{\text {}} \mathrm{P}<0.05$ vs. control; ${ }^{\mathrm{P}} \mathrm{P}<0.05$ vs. OGD+vehicle.

we determined the phosphorylated/total ERK protein ratio. As shown in Fig. 6, OGD significantly increased the level of activated ERK $(\mathrm{P}<0.05)$, and this effect was reversed by resveratrol at concentrations of 50 and $100 \mu \mathrm{M}(\mathrm{P}<0.05)$. This result suggests that ERK plays an important role in OGD-induced cell apoptosis.

Resveratrol inhibits OGD-induced MMP-9 expression and cell apoptosis via ERK1/2. To investigate the pathway via which resveratrol exerts neuroprotective effects, we studied the effect of resveratrol on the protein level of activated ERK, that of MMP-9 and of proteins related to cell apoptosis. Cells were pretreated with U0126 $15 \mathrm{~min}$ prior to resveratrol treatment. Resveratrol was added to the cultures at a final concentration of $50 \mu \mathrm{M}$. Untreated cultures received an equivalent volume of carrier solvent $(0.1 \%$ DMSO). The duration of the treatment was from OGD until the end of the experiment. U0126 treatment inhibited MMP-9 expression and activation (cleavage) of caspase-3, as well as Bax expression, but promoted the expression of the anti-apoptotic molecule Bcl-2 (Fig. 7). The level of activated ERK (p-ERK) was reduced by U0126 treatment. This suggests that resveratrol inhibits MMP-9 expression and cell apoptosis via attenuating the activation of ERK1/2.

\section{Discussion}

Stroke annually causes brain injury in millions of individuals worldwide. However, there is currently no approved therapy that can reduce infarction size or neurological disability $(21,22)$. An altered blood supply in the brain (ischemia) deprives brain cells of glucose and oxygen, causing irreversible brain damage within minutes. The brain is particularly vulnerable

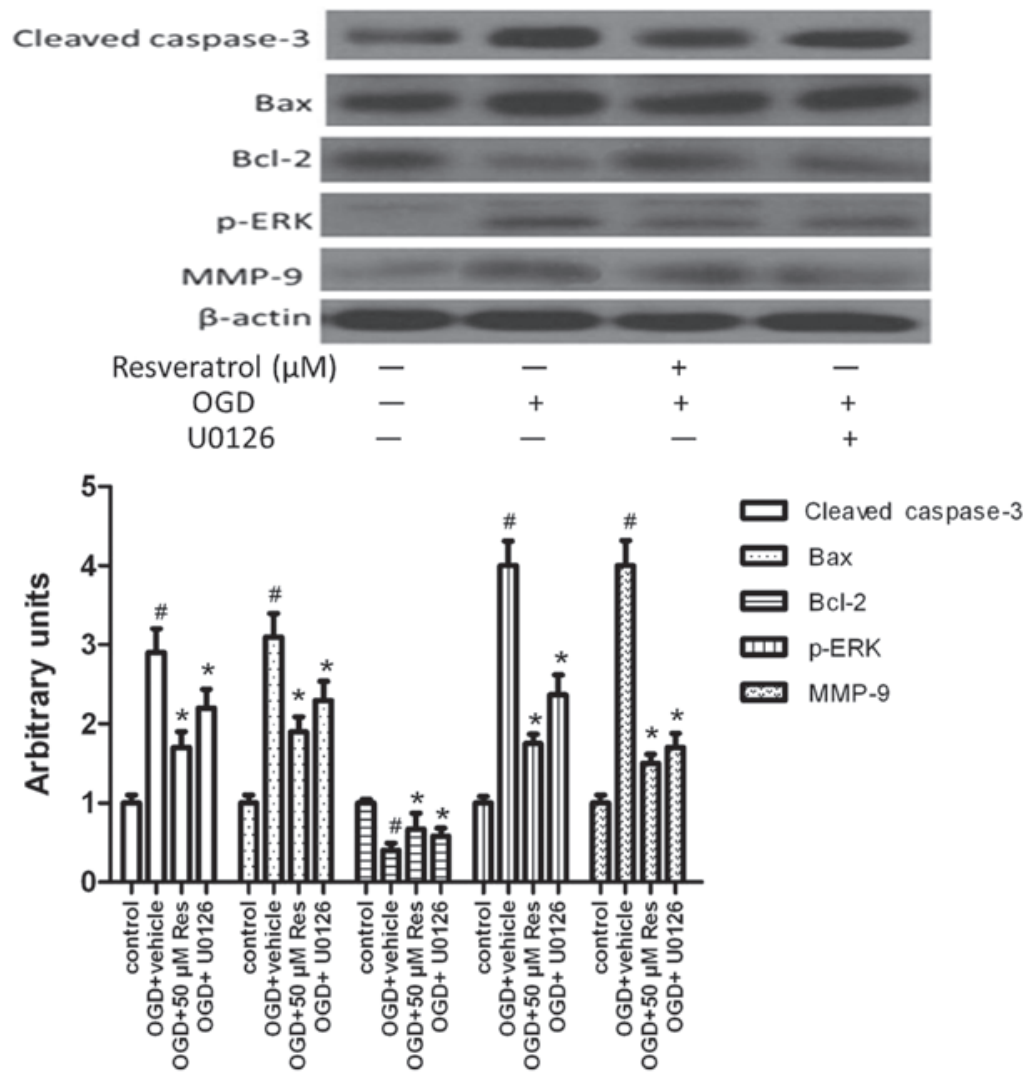

Figure 7. Oxygen-glucose deprivation (OGD) activates extracellular signal-regulated kinase (ERK)1/2 and the expression of proteins related to cell apoptosis, and these effects are reverted by resveratrol and the ERK innhibitor U0126. The protein levels of matrix metalloproteinase-9 (MMP-9), phosphorylated (p)-ERK, Bcl-2, cleaved caspase-3 and Bax were determined using western blotting and the corresponding antibodies. $\beta$-actin was used as an internal control. Each blot is representative of three independent experiments. ${ }^{\#} \mathrm{P}<0.05$ vs. control; ${ }^{*} \mathrm{P}<0.05$ vs. OGD+vehicle. Res, resveratrol. 
to ischemia due to: i) the very high rate of oxidative metabolism in this organ, requiring a continuous supply of oxygen and glucose; ii) the metabolic interdependence of neurons and astrocytes, two types of brain cells; and iii) the sensitivity of neurons to disruptions in ion homeostasis caused by ischemia (23). Although ischemia is a well-recognized cause of cellular damage, the underlying mechanism is not fully understood.

It has been reported that an antioxidant which has a neuroprotective effect in ischemic brain injury is involved in neuronal apoptosis (24). Apoptosis occurs in vulnerable neurons, such as the cortex and the hippocampus, during early reperfusion (25). Resveratrol shows biological and pharmacological effects on cells, including anti-oxidant, -inflammatory, -mutagenic, -carcinogenic and -apoptotic (26).

Previous reports highlighted MMP-9 as a key factor in brain injury following cerebral ischemia reperfusion, and suggested that this protein may represent a new therapeutic target $(27,28)$. We previously verified that resveratrol has neuroprotective effects and can reduce the elevated level of MMP-9 induced by cerebral ischemia-reperfusion in a murine model of transient middle cerebral artery occlusion (17). Both the total protein and RNA levels of MMP-9 were examined in cerebral tissue. MMP-9 is a key regulator of apoptosis of hypertrophic chondrocytes, and null mutations in the gene can delay apoptosis (16). MMP-9 controls Schwann cell proliferation and phenotypic remodeling via IGF-1 and ErbB receptor-mediated activation of the MEK/ERK pathway (29).

We examined the viability of cells subjected to transient OGD for different periods in pilot MTT experiments, and found that a 3-h OGD followed by $21 \mathrm{~h}$ of reoxygenation caused death in $~ 50 \%$ of the cells and was the most suitable condition for establishing stable ischemia in vitro. Resveratrol is a polyphenolic compound. It can be fully dissolved in DMSO at high densities and stored at $-80^{\circ} \mathrm{C}$ for a long period. The highest dose of resveratrol used in our study was $100 \mu \mathrm{M}$, containing $0.1 \%$ DMSO. Pilot experiments showed that 0.01-0.2\% DMSO has no harmful effects on cells when added to the culture medium alone, which allowed to exclude potential confounding effects of this solvent on the results obtained from resveratrol treatment. Therefore, the cell viability assay demonstrated that resveratrol treatment has protective effects against transient OGD in primary cortical neuron cultures.

In the present study, we investigated the protective effects of resveratrol at the cellular level by studying its anti-apoptotic effect and the effect on expression of MMP-9 in both vehicleand resveratrol-treated primary cortical neuron cultures. We found that OGD insult caused a marked increase in the percentage of apoptotic cells (51\%), which was reduced by the addition of resveratrol in a dose-dependent manner. Results of western blot analysis showed that resveratrol decreased the high level of the MMP-9 protein (105 kDa), which was induced by transient OGD. The most significant results were observed at the 50-100 $\mu \mathrm{M}$ doses.

Caspases play an important role in the apoptotic process. Caspase-3 activates the DNA fragmentation factor, which in turn activates endonucleases to cleave nuclear DNA and ultimately leads to cell death (30). In the present study, OGD led to an increase in caspase-3 activity, but resveratrol treatment effectively inhibited the activation of caspase-3.
Bcl-2 is a member of the anti-apoptotic Bcl-2 family, which plays a key role in regulating mitochondrial-mediated apoptotic cell death and can attenuate caspase- 3 activation $(31,32)$. In the present study, OGD induced a decrease in the Bcl-2 protein level in cortical cells, whereas Bcl-2 expression was increased in cells treated with resveratrol. The Bax protein belongs to the $\mathrm{Bcl}-2$ gene family and can repress the function of $\mathrm{Bcl}-2$ by forming a dimer with Bcl-2. OGD induced the expression of Bax, the level of which was effectively suppressed following treatment with resveratrol.

ERK activation controls various cell responses, such as proliferation, migration, differentiation, and death (33). Resveratrol potently and efficiently inhibits ERK signaling in sensory neurons in vitro (34). We found that resveratrol can inhibit the activation of ERK in a dose-dependent manner. In addition, we explored the relationship between resveratrol, ERK, MMP-9 and cell apoptosis. U0126, a specific inhibitor of ERK, was added to cortical cultures and was found to have a similar effect on ERK activation, MMP-9 and cell apoptosis to that exerted by resveratrol.

Overall, our results indicate that OGD induces apoptosis through regulating canonical apoptosis signaling and the expression of MMP-9; the anti-apoptotic effect of resveratrol, along with the inhibition of MMP-9 expression it causes contribute to the suppression of ERK activation. The results reported here further support that resveratrol, contained in red wine and other natural products, has neuroprotective effects in cerebral ischemia. Thus, resveratrol may be considered a suitable candidate drug for stroke treatment.

\section{Acknowledgements}

This study was supported by a research grant from the National Natural Science Fund of China (no. 30901553).

\section{References}

1. Celotti E, Ferrarini R, Zironi R, et al: Resveratrol content of some wines obtained from dried Valpolicella grapes: Recioto and Amarone. J Chromatogr A 730: 47-52, 1996.

2. Pany S, Majhi A and Das J: PKC activation by resveratrol derivatives with unsaturated aliphatic chain. PLoS One 7: e52888, 2012.

3. Das J, Pany S and Majhi A: Chemical modifications of resveratrol for improved protein kinase $\mathrm{C}$ alpha activity. Bioorg Med Chem 19: 5321-5333, 2011.

4. Kesherwani V, Atif F, Yousuf S, et al: Resveratrol protects spinal cord dorsal column from hypoxic injury by activating Nrf-2. Neuroscience 241: 80-88, 2013.

5. Huang SS, Tsai MC, Chih CL, et al: Resveratrol reduction of infarct size in Long-Evans rats subjected to focal cerebral ischemia. Life Sci 69: 1057-1065, 2001.

6. Wang Q, Xu J, Rottinghaus GE, et al: Resveratrol protects against global cerebral ischemic injury in gerbils. Brain Res 958: 439-447, 2002.

7. Doyle GA, Pierce RA and Parks WC: Transcriptional induction of collagenase-1 in differentiated monocyte-like (U937) cells is regulated by AP-1 and an upstream C/EBP-beta site. J Biol Chem 272: 11840-11849, 1997.

8. Lu X, Xu H, Sun B, et al: Enhanced neuroprotective effects of resveratrol delivered by nanoparticles on hydrogen peroxide-induced oxidative stress in rat cortical cell culture. Mol Pharm 10: 2045-2053, 2013.

9. de la Torre E, Hovsepian E, Penas FN, et al: Macrophages derived from septic mice modulate nitric oxide synthase and angiogenic mediators in the heart. J Cell Physiol 228: 1584-1593, 2013. 
10. Pfefferkorn T and Rosenberg GA: Closure of the blood-brain barrier by matrix metalloproteinase inhibition reduces rtPA-mediated mortality in cerebral ischemia with delayed reperfusion. Stroke 34: 2025-2030, 2003.

11. Dufour A and Overall CM: Missing the target: matrix metalloproteinase antitargets in inflammation and cancer. Trends Pharmacol Sci 34: 233-242, 2013.

12. Saarialho-Kere UK, Welgus HG and Parks WC: Distinct mechanisms regulate interstitial collagenase and $92-\mathrm{kDa}$ gelatinase expression in human monocytic-like cells exposed to bacterial endotoxin. J Biol Chem 268: 17354-17361, 1993.

13. Nagaoka I and Hirota S: Increased expression of matrix metalloproteinase-9 in neutrophils in glycogen-induced peritoneal inflammation of guinea pigs. Inflamm Res 49: 55-62, 2000.

14. Nguyen M, Arkell J and Jackson CJ: Human endothelial gelatinases and angiogenesis. Int J Biochem Cell Biol 33: 960-970, 2001.

15. Cayabyab FS, Gowribai K and Walz W: Involvement of matrix metalloproteinases-2 and -9 in the formation of a lacuna-like cerebral cavity. J Neurosci Res 91: 920-933, 2013.

16. Vu TH, Shipley JM, Bergers G, et al: MMP-9/gelatinase B is a key regulator of growth plate angiogenesis and apoptosis of hypertrophic chondrocytes. Cell 93: 411-422, 1998.

17. Gao D, Zhang X, Jiang X, et al: Resveratrol reduces the elevated level of MMP-9 induced by cerebral ischemia-reperfusion in mice. Life Sci 78: 2564-2570, 2006.

18. Tauskela JS, Comas T, Hewitt K, et al: Cross-tolerance to otherwise lethal N-methyl-D-aspartate and oxygen-glucose deprivation in preconditioned cortical cultures. Neuroscience 107: 571-584, 2001

19. Gong QH, Wang Q, Shi JS, et al: Inhibition of caspases and intracellular free $\mathrm{Ca}^{2+}$ concentrations are involved in resveratrol protection against apoptosis in rat primary neuron cultures. Acta Pharmacol Sin 28: 1724-1730, 2007.

20. Cagnol S and Chambard JC: ERK and cell death: mechanisms of ERK-induced cell death - apoptosis, autophagy and senescence. FEBS J 277: 2-21, 2010.

21. Schaller B and Graf R: Cerebral ischemia and reperfusion: the pathophysiologic concept as a basis for clinical therapy. J Cereb Blood Flow Metab 24: 351-371, 2004.
22. Moskowitz MA, Lo EH and Iadecola C: The science of stroke: mechanisms in search of treatments. Neuron 67: 181-198, 2010.

23. Hayashi $\mathrm{T}$ and Abe K: Ischemic neuronal cell death and organellae damage. Neurol Res 26: 827-834, 2004.

24. Fujimura M, Tominaga T and Chan PH: Neuroprotective effect of an antioxidant in ischemic brain injury: involvement of neuronal apoptosis. Neurocrit Care 2: 59-66, 2005.

25. Krause GS, White BC, Aust SD, et al: Brain cell death following ischemia and reperfusion: a proposed biochemical sequence. Crit Care Med 16: 714-726, 1988.

26. Lee MK, Kang SJ, Poncz M, et al: Resveratrol protects SH-SY5Y neuroblastoma cells from apoptosis induced by dopamine. Exp Mol Med 39: 376-384, 2007.

27. Magnoni S, Baker A, George SJ, et al: Differential alterations in the expression and activity of matrix metalloproteinases 2 and 9 after transient cerebral ischemia in mice. Neurobiol Dis 17: 188-197, 2004.

28. Deng X, Zhong Y, Gu L, et al: MiR-21 involve in ERK-mediated upregulation of MMP9 in the rat hippocampus following cerebral ischemia. Brain Res Bull 94: 56-62, 2013

29. Chattopadhyay S and Shubayev VI: MMP-9 controls Schwann cell proliferation and phenotypic remodeling via IGF-1 and ErbB receptor-mediated activation of MEK/ERK pathway. Glia 57: 1316-1325, 2009

30. Wang XJ and Xu JX: Salvianic acid A protects human neuroblastoma $\mathrm{SH}-\mathrm{SY} 5 \mathrm{Y}$ cells against $\mathrm{MPP}^{+}$-induced cytotoxicity. Neurosci Res 51: 129-138, 2005.

31. Sawada M, Nakashima S, Banno Y, et al: Influence of Bax or Bcl-2 overexpression on the ceramide-dependent apoptotic pathway in glioma cells. Oncogene 19: 3508-3520, 2000.

32. Yamakawa $\mathrm{H}$, Ito $\mathrm{Y}$, Naganawa $\mathrm{T}$, et al: Activation of caspase- 9 and -3 during $\mathrm{H}_{2} \mathrm{O}_{2}$-induced apoptosis of PC12 cells independent of ceramide formation. Neurol Res 22: 556-564, 2000.

33. Murphy LO and Blenis J: MAPK signal specificity: the right place at the right time. Trends Biochem Sci 31: 268-275, 2006.

34. Tillu DV, Melemedjian OK, Asiedu MN, et al: Resveratrol engages AMPK to attenuate ERK and mTOR signaling in sensory neurons and inhibits incision-induced acute and chronic pain. Mol Pain 8: 5, 2012. 